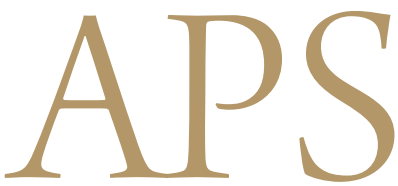

Archives of Plastic Surgery

\title{
Optical Biopsy of Peripheral Nerve Using Confocal Laser Endomicroscopy: A New Tool for Nerve Surgeons?
}

\author{
Christopher S Crowe ${ }^{1}$, Joseph C Liao ${ }^{2}$, Catherine M Curtin ${ }^{1}$ \\ ${ }^{1}$ Division of Plastic and Reconstructive Surgery, ${ }^{2}$ Division of Urology, Stanford School of Medicine, Stanford, CA, USA
}

Peripheral nerve injuries remain a challenge for reconstructive surgeons with many patients obtaining suboptimal results. Understanding the level of injury is imperative for successful repair. Current methods for distinguishing healthy from damaged nerve are time consuming and possess limited efficacy. Confocal laser endomicroscopy (CLE) is an emerging optical biopsy technology that enables dynamic, high resolution, sub-surface imaging of live tissue. Porcine sciatic nerve was either left undamaged or briefly clamped to simulate injury. Diluted fluorescein was applied topically to the nerve. CLE imaging was performed by direct contact of the probe with nerve tissue. Images representative of both damaged and undamaged nerve fibers were collected and compared to routine H\&E histology. Optical biopsy of undamaged nerve revealed bands of longitudinal nerve fibers, distinct from surrounding adipose and connective tissue. When damaged, these bands appear truncated and terminate in blebs of opacity. H\&E staining revealed similar features in damaged nerve fibers. These results prompt development of a protocol for imaging peripheral nerves intraoperatively. To this end, improving surgeons' ability to understand the level of injury through real-time imaging will allow for faster and more informed operative decisions than the current standard permits.

Keywords Trauma, nervous system / Microscopy / Biopsy / Diagnostic imaging / Histological techniques

\author{
Correspondence: \\ Christopher S Crowe \\ Stanford School of Medicine, \\ Department of Surgery, Division of \\ Plastic and Reconstructive Surgery, \\ 770 Welch Road, Suite 400, Palo Alto, \\ CA 94304, USA \\ Tel: +1-650-723-5256 \\ Fax: +1-650-721-3422 \\ E-mail: cscrowe@stanford.edu
}

No potential conflict of interest relevant to this article was reported.

Received: 27 Apr $2015 \bullet$ Revised: 11 Jun $2015 \bullet$ Accepted: 19 Jun 2015

pISSN: 2234-6163 • elSSN: 2234-6171 • http://dx.doi.org/10.5999/aps.2015.42.5.626• Arch Plast Surg 2015;42:626-629

\section{INTRODUCTION}

Peripheral nerve injuries present a considerable challenge for reconstructive surgeons. Great strides have been made over the last thirty years, improving both our understanding of the biology of nerve regeneration and surgical repair techniques. Yet there are still obstacles to overcome with far too many patients still experiencing permanent loss of function despite aggressive treatment [1].
Recovery after nerve repair can be influenced by the duration of injury, patient factors, and type of injury [2]. One critical component for optimizing outcomes is the surgeon's understanding of the extent of nerve injury. When a patient presents with a stretch nerve injury, for example, it can be difficult for the surgeon to delineate where the injured nerve ends and healthy nerve begins. The injured area is regularly surrounded by scar, making the assessment of how well axons are regenerating a challenging task. Currently surgeons use a combination of phys- 
ical exam, electro-diagnostics, and intraoperative monitoring before deciding the correct operative intervention. Nevertheless, the surgeon still needs to use clinical judgment and maneuvers, such as slicing the nerve sequentially like a loaf of bread until healthy-appearing axons are encountered. Another tool to identify the level of intact axons could reduce the uncertainty about when, where and what to nerve graft.

\section{IDEA}

Confocal laser endomicroscopy (CLE) is an optical biopsy technology that enables dynamic, high resolution, sub-surface imaging of live tissue [3]. An imaging probe, which emits a lowpower $488 \mathrm{~nm}$ laser, is held in direct contact with the surface of the tissue for image acquisition. Fluorescein, a Food and Drug Administration approved drug, is used as the contrast agent and can be safely administered either intravenously or topically. Probes with varying diameters ( 0.85 to $2.6 \mathrm{~mm}$ ) and optical specifications are available. The technology has been applied endoscopically in gastroenterology to detect areas of dysplasia in the colon [4-6], stomach [7,8], and esophagus [9-11]. An additional application is found in urology, in which CLE is used to image bladder cancer during cystoscopy [12]. The goal of this pilot study is to assess the feasibility of CLE to image the internal architecture of nerve in a tissue-preserving manner.

Fresh porcine sciatic nerve was obtained commercially (innovative technologies) and sectioned into segments approximately $1.5 \mathrm{~cm}$ in length and $0.5 \mathrm{~cm}$ in diameter. For the purposes of distinguishing damaged from undamaged nerve fibers, a straight 5-inch Halsted mosquito was clamped over nerve and locked for 30 seconds. Subsequently, a $0.05 \%$ solution of fluorescein was topically applied to the surface of the both damaged and undamaged nerve using a cotton-tipped swab. After 6-minute incubation, the nerve was rinsed with $15 \mathrm{~mL}$ normal saline. CLE was performed using a 2.6-mm diameter fiber-optic imaging probe (Cellvizio, Mauna Kea Technologies Inc., Suwanee, GA, USA) with the following optical specifications: spatial resolution $1 \mu \mathrm{m}$, field of view $240 \mu \mathrm{m}$, depth of view $60 \mu \mathrm{m}$, and optical slice thickness $10 \mu \mathrm{m}$. For image acquisition, the imaging probe was held in direct en face contact with the tissue (Fig. 1). While the depth of view is fixed, different optical sections may be obtained by varying the pressure of the probe against the surface of the nerve. Images were recorded as video sequences at 30 frames per second. For image processing and editing, Cellvizio software was used. After imaging, the nerve was fixed and standard hematoxylin and eosin (H\&E) histology was performed (Histotec). Representative confocal images were compared with histopathology.

\section{Fig. 1. Application of CLE probe to target tissue}

Direct application of the imaging probe to tissue allows for dynamic, real time visualization of internal, sub-surface architecture. CLE, confocal laser endomicroscopy.

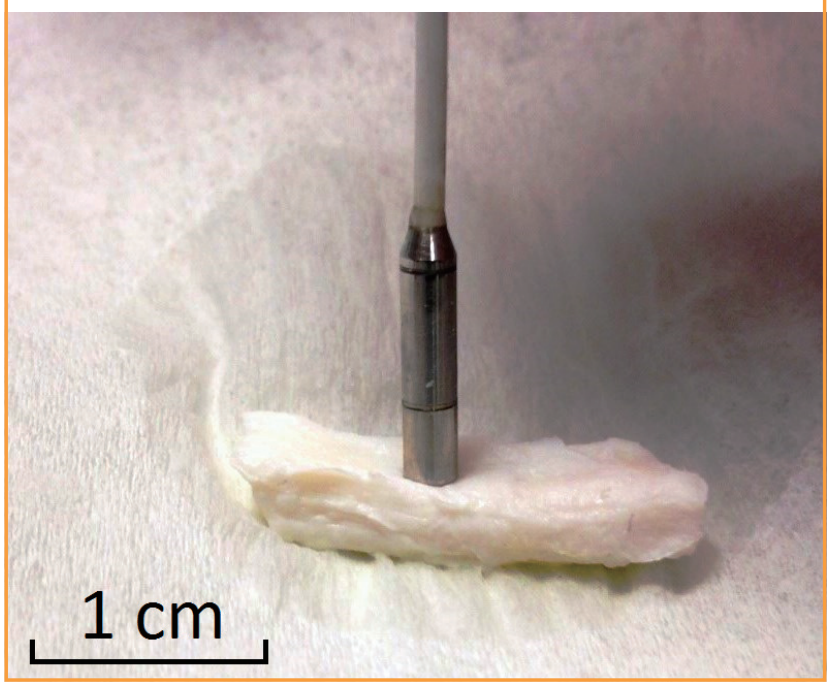

Fig. 2A depicts a representative ex vivo image of undamaged porcine sciatic nerve showing longitudinal thin dark bands consistent with nerve fibers. Corresponding H\&E histology shows the similar banding pattern of the nerve tissue (Fig. 2D). Undamaged nerve was successfully distinguished from damaged nerve fibers. Clamped nerve segments displayed disrupted fascicles (Fig. 2B), which prematurely terminate into areas of opacity. These regions likely represent areas of pocketing due to the application of the clamp, allowing blebs of fluorescein to accumulate within nerve tissue. Corresponding histology shows a similar disruption of nerve fascicles (Fig. 2E). Longitudinal nerve fibers were also distinct from surrounding adipocytes, characterized by large ovoid cells with abundant cytoplasm (Fig. 2C), similar to our previous in vivo imaging findings of adipocytes in humans [11]. Corresponding H\&E histology for adipocytes (Fig. 2F) is also shown for comparison.

\section{DISCUSSION}

The reconstructive nerve surgeon is charged with three primary tasks when evaluating a patient who presents with a nerve injury. The first is to assess the location, severity, and extent of neuronal damage. The second is deciding if and when to bring the patient to the operating theater for nerve repair. Lastly, if surgery is warranted, the nerve surgeon must decide which treatment best fits the injury. This task relies on intraoperative assessment and would benefit from a new innovation to visualize the microarchitecture of peripheral nerve.

Success of nerve surgery is dependent upon the surgeon find- 


\section{Fig. 2. Optical biopsy using CLE and corresponding histology}

Using CLE, parallel nerve fascicles can be observed in undamaged specimens (A), appearing distinct from the disrupted fascicles of damaged nerve that terminate in areas marked by increased opacity (red arrows) (B), and surrounding adipose tissue (C). Corresponding H\&E histology is shown for comparison ( $D, E, F)$ with similar disruption of fascicular continuity (red arrows) in damaged nerve (D) $\left(H \& E_{1} \times 400\right)$. CLE, confocal laser endomicroscopy.
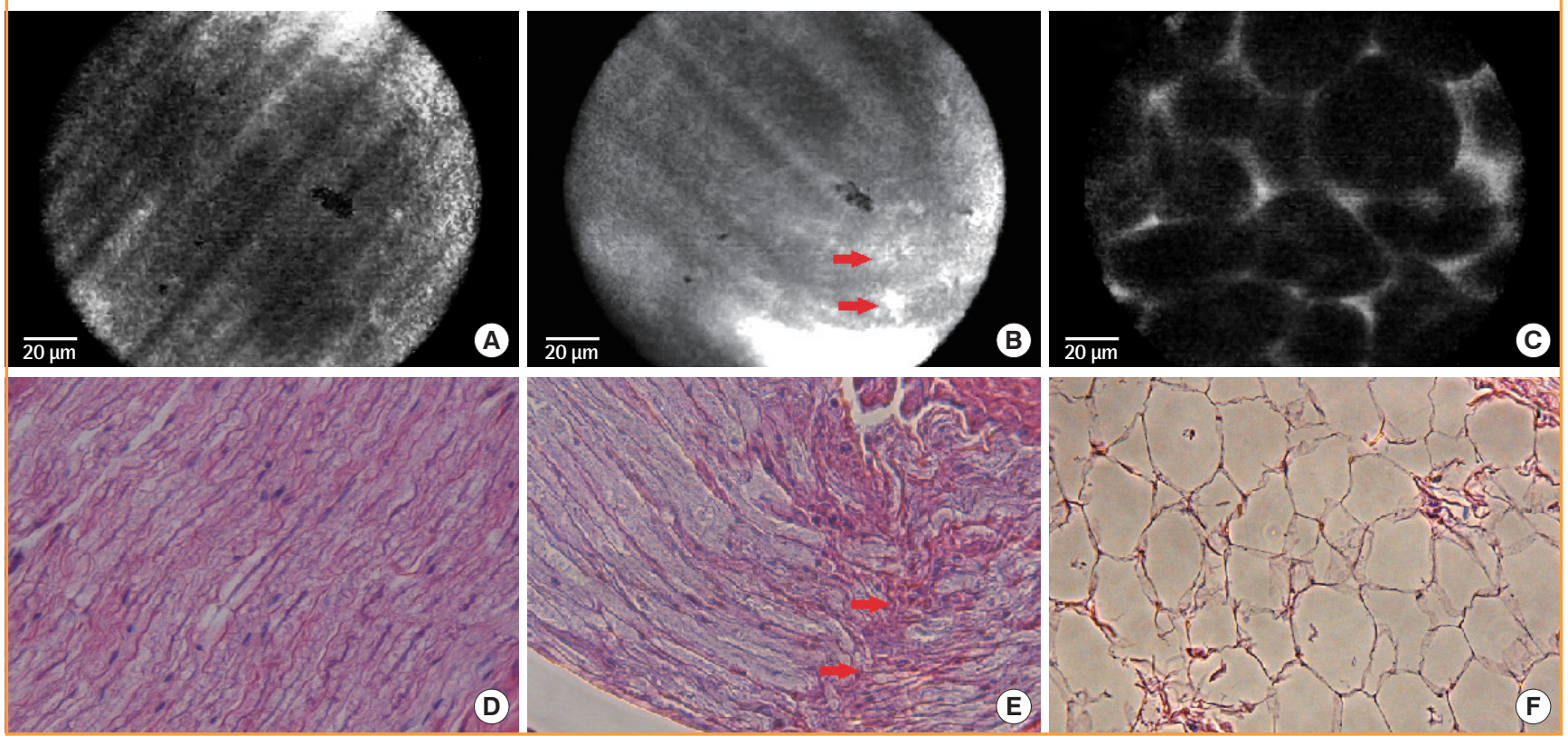

ing and removing damaged nerve until the "zone of injury" becomes well defined. This remains a challenging undertaking, especially in cases of crush and avulsion injuries. Reconstructive nerve surgeons aim to resect as little tissue as possible while still achieving coaptation between healthy nerve fibers. In current practice, no tissue-preserving methods for determining the location of healthy tissue exist as an adjunct to progressive resection. This pilot study demonstrates the feasibility of CLE for distinguishing damaged from undamaged porcine nerve tissue with discernible features comparable to standard histology.

CLE has already found surgical utility for the purposes of visualizing and demarcating areas of gastroenterologic and urologic dysplasia. Monitoring systems are portable and can readily be employed in the operating theater. Probe tips are compact and flexible, capable of fitting through even the smallest incisions. Probes can be sterilized in the usual fashion and reused multiple times without affecting resolution.

CLE could readily be incorporated into the current standard treatment protocol for nerve repair without significantly prolonging operative time. Once proper exposure of the damaged nerve is achieved, fluorescein would be applied directly while further dissection is pursued. After a brief incubation period, the nerve would be rinsed with normal saline. The sterile probe tip would then be applied directly to the nerve and real-time image would be generated. These images could help the surgeon delineate healthy and injured fibers prior to resection.

Our next step is to perform CLE intraoperatively, evaluating the ability of CLE to distinguish healthy from injured nerve in patients. Our hope is that by improving surgeons' ability to understand the level of injury through real-time imaging, faster and more informed operative decisions could be made than the current standard permits. The clear benefit of imaging with CLE is that it can be performed concurrently with routine diagnostics without lengthening a procedure or placing the patient at additional risk.

As more studies proving the efficacy of CLE emerge in a variety of surgical disciplines, it becomes likely that even smaller medical centers will find the equipment cost effective. The device only has to be purchased once, and additional probes can be used for a range of different diagnostic purposes. We are optimistic that advancements in peripheral nerve imaging will translate into better care for trauma patients recovering from nerve injury.

\section{REFERENCES}

1. Lundborg G. A 25-year perspective of peripheral nerve surgery: evolving neuroscientific concepts and clinical significance. J Hand Surg Am 2000;25:391-414.

2. Sunderland S. Nerve injuries and their repair: a critical ap- 
praisal. Edinburgh: Churchill Livingstone; 1991.

3. Meining A. Confocal endomicroscopy. Gastrointest Endosc Clin N Am 2009; 19:629-35.

4. Kiesslich R, Burg J, Vieth M, et al. Confocal laser endoscopy for diagnosing intraepithelial neoplasias and colorectal cancer in vivo. Gastroenterology 2004;127:706-13.

5. Wang TD, Friedland S, Sahbaie P, et al. Functional imaging of colonic mucosa with a fibered confocal microscope for real-time in vivo pathology. Clin Gastroenterol Hepatol 2007;5:1300-5.

6. Gomez V, Buchner AM, Dekker E, et al. Interobserver agreement and accuracy among international experts with probe-based confocal laser endomicroscopy in predicting colorectal neoplasia. Endoscopy 2010;42:286-91.

7. Banno K, Niwa Y, Miyahara R, et al. Confocal endomicroscopy for phenotypic diagnosis of gastric cancer. J Gastroenterol Hepatol 2010;25:712-8.

8. Li WB, Zuo XL, Zuo F, et al. Characterization and identifi- cation of gastric hyperplastic polyps and adenomas by confocal laser endomicroscopy. Surg Endosc 2010;24:517-24.

9. Dunbar KB, Okolo P 3rd, Montgomery E, et al. Confocal laser endomicroscopy in Barrett's esophagus and endoscopically inapparent Barrett's neoplasia: a prospective, randomized, double-blind, controlled, crossover trial. Gastrointest Endosc 2009; 70:645-54.

10. Pech O, Rabenstein T, Manner H, et al. Confocal laser endomicroscopy for in vivo diagnosis of early squamous cell carcinoma in the esophagus. Clin Gastroenterol Hepatol 2008;6:89-94.

11. Kiesslich R, Gossner L, Goetz M, et al. In vivo histology of Barrett's esophagus and associated neoplasia by confocal laser endomicroscopy. Clin Gastroenterol Hepatol 2006;4: 979-87.

12. Wu K, Liu JJ, Adams W, et al. Dynamic real-time microscopy of the urinary tract using confocal laser endomicroscopy. Urology 2011;78:225-31. 